\title{
Sources of variability in estimates of the prevalence of Alzheimer's disease in the United States
}

\author{
Robert S. Wilson ${ }^{\mathrm{a}, \mathrm{b}, \mathrm{c}, *}$, David R. Weir ${ }^{\mathrm{d}}$, Sue E. Leurgans ${ }^{\mathrm{b}}$, Denis A. Evans ${ }^{\mathrm{e}, \mathrm{f}}$, \\ Liesi E. Hebert ${ }^{\mathrm{e}, \mathrm{f}}$, Kenneth M. Langa ${ }^{\mathrm{d}, \mathrm{g}, \mathrm{h}}$, Brenda L. Plassman ${ }^{\mathrm{i}}$, \\ Brent J. Small ${ }^{\mathrm{j}}$, David A. Bennett $\mathrm{t}^{\mathrm{a}, \mathrm{b}}$ \\ ${ }^{a}$ Rush Alzheimer's Disease Center, Rush University Medical Center, Chicago, IL, USA \\ ${ }^{b}$ Department of Neurological Sciences, Rush University Medical Center, Chicago, IL, USA \\ ${ }^{c}$ Department of Behavioral Sciences, Rush University Medical Center, Chicago, IL, USA \\ ${ }^{d}$ Institute for Social Research, University of Michigan, Ann Arbor, MI, USA \\ ${ }^{e}$ Rush Institute for Healthy Aging, Rush University Medical Center, Chicago, IL, USA \\ ${ }^{f}$ Department of Internal Medicine, Rush University Medical Center, Chicago, IL, USA \\ ${ }^{g}$ Division of General Medicine, Department of Medicine, School of Medicine, University of Michigan, Ann Arbor, MI, USA \\ ${ }^{h}$ Veterans Affairs Center for Practice Management and Outcomes Research, Ann Arbor, MI, USA \\ ${ }^{i}$ Department of Psychiatry and Behavioral Sciences, Duke University Medical Center, Durham, NC, USA \\ ${ }^{j}$ School of Aging Studies, University of South Florida, Tampa, FL, USA
}

\begin{abstract}
Background: The prevalence of Alzheimer's disease (AD) in the United States was estimated at 2.3 million in 2002 by the Aging, Demographics, and Memory Study (ADAMS), which is almost 50\% less than the estimate of 4.5 million in 2000 derived from the Chicago Health and Aging Project. Methods: We considered how differences in diagnostic criteria may have contributed to these differences in AD prevalence.

Results: We identified several important differences in diagnostic criteria that may have contributed to the differing estimates of AD prevalence. Two factors were especially noteworthy. First, the Diagnostic and Statistical Manual of Mental Disorders III-R and IV criteria of functional limitation documented by an informant used in ADAMS effectively concentrated the diagnosis of dementia toward a relatively higher level of cognitive impairment. ADAMS separately identified a category of cognitive impairment not dementia and within that group there were a substantial number of cases with "prodromal" AD (a maximum of 1.95 million with upweighting). Second, a substantial proportion of dementia in ADAMS was attributed to either vascular disease (representing a maximum of 0.59 million with upweighting) or undetermined etiology (a maximum of 0.34 million), whereas most dementia, including mixed dementia, was attributed to AD in the Chicago Health and Aging Project. Conclusion: The diagnosis of $\mathrm{AD}$ in population studies is a complex process. When a diagnosis of $\mathrm{AD}$ excludes persons meeting criteria for vascular dementia, when not all persons with dementia are assigned an etiology, and when a diagnosis of dementia requires an informant report of functional limitations, the prevalence is substantially lower and the diagnosed cases most likely have a relatively higher level of impairment.
\end{abstract}

(C) 2011 The Alzheimer's Association. All rights reserved.

Keywords: $\quad$ Epidemiologic studies; Dementia; Alzheimer's disease; Vascular dementia; Mild cognitive impairment; Cognitive impairment no dementia

The authors have no conflicts to disclose. The sponsors had neither a role in the analysis or interpretation of these data, nor in the content of the article. Appropriate approval procedures were used concerning human subjects.

*Corresponding author.

E-mail address: rwilson@rush.edu

\section{Introduction}

Alzheimer's disease (AD) is the leading cause of dementia in old age. Because of its devastating effect on cognition and other behaviors and its chronic course, the disease poses 
enormous public health problems. These problems are projected to increase in the coming decades with the aging of the U.S. population [1-3], thus underscoring the urgency of developing strategies to prevent the disease or delay the onset of its behavioral symptoms and associated costs.

Knowledge of disease prevalence is critical for public health planning and policy. Most estimates of the prevalence of $\mathrm{AD}$ in the U.S. population are based on a single community or group of communities [1,3-5]. By contrast, Plassman et al [6] in the Aging, Demographics, and Memory Study (ADAMS) used data from the ongoing Health and Retirement Study [7] to identify a nationally representative sample of older persons for detailed clinical evaluation. They estimated that, in 2002, there were 2.3 million people in the United States with AD. This estimate is comparable with recent estimates from systematic reviews of the dementia prevalence literature $[8,9]$. However, the ADAMS estimate is only about one-half of the 4.5 million affected individuals estimated by Hebert et al [3] for the year 2000 using data from the Chicago Health and Aging Project (CHAP). In this article, we consider factors that may have contributed to the differences between these two estimates of the prevalence of AD in the United States, and use ADAMS data to provide some evidence on the cognitive, functional, and genetic characteristics of groups whose diagnostic status would likely differ between the two study protocols.

\section{Overview of study differences}

The source studies from which the Hebert et al [3] and Plassman et al [6] AD prevalence estimates were derived each involved taking a stratified random sample of older persons from a defined population, inviting them to undergo a uniform evaluation to support clinical classification of dementia and $\mathrm{AD}$, and then using census data to upweight the results to the U.S. population. Because each study involved estimating disease prevalence in millions on the basis of examination of less than 1000 individuals, some difference between the studies would be expected because of random error, even when identical methods are used. However, the size of the observed difference suggests that other factors were involved. There were several methodological differences between the source studies with the potential to affect $\mathrm{AD}$ prevalence estimates. We begin by noting several differences in methods that might, in theory, contribute to differences in estimates but that are likely to be of minor importance. We then focus on two issues that we think might account for most of the difference.

\subsection{National representation}

The ADAMS sample was drawn from a nationally representative cohort, whereas the CHAP samples were drawn from a single urban community that was not representative of the United States. To the extent that there is regional var- iation in $\mathrm{AD}$ prevalence, ADAMS should provide a more accurate estimate.

\subsection{Participation rate}

In ADAMS, 56\% of the nondeceased target sample completed the clinical evaluation as compared with $75 \%$ in the most comparable clinical evaluation in CHAP. Because nonparticipation is associated with poorer cognitive performance in some studies, one might be concerned that lower participation in ADAMS could have led to an underestimation of dementia prevalence, as reported by Plassman et al [6]. However, the ADAMS investigators conducted a thorough investigation and found no association between cognitive ability as measured in the Health and Retirement Study (HRS) either before or after the ADAMS assessment and participation in ADAMS. They also used propensity analyses to adjust for potential bias using data available from the parent HRS. Therefore, in this case, the overall effect of differential participation on results is likely to be small.

\subsection{Age}

The ADAMS estimate of AD prevalence was for individuals of age $\geq 71$ years, whereas CHAP included 65 - to 70 -year-olds as well as older individuals. However, only an estimated 26,000 of the 4.5 million individuals in the CHAP estimate were in the age range of 65 to 70 years. Therefore, the slight difference at the lower end of the age ranges studied is unlikely to have substantially contributed to the differential estimates of disease prevalence.

\subsection{Estimating prevalence from incidence data}

Another difference underlying the two estimates of $\mathrm{AD}$ prevalence is that the estimate of Hebert et al [3] was based on $\mathrm{AD}$ incidence in the source study (CHAP), whereas that of Plassman et al [6] was based on AD prevalence in the source study (ADAMS). Prevalence projections based on incidence studies can overstate prevalence if there is overdiagnosis in the assessment because false positives in the follow-up wave would inflate the incidence rates, whereas overdiagnosis for the calculation of mortality ratios would increase estimated survival of individuals with $\mathrm{AD}$ in the projections. Prevalence studies can understate prevalence if they underdiagnose disease or if they underestimate persons with rapidly progressive disease and death [10]. There is no evidence that these concerns are of substantial quantitative importance in this comparison.

\section{Possible modifiers of prevalence estimates}

In this section, we consider further differences between the source studies used to estimate AD prevalence. In addition, we provide a quantitative estimate of the effect of each factor on $\mathrm{AD}$ prevalence. 


\subsection{Diagnostic criteria for dementia}

Clinical classification of $\mathrm{AD}$ requires that dementia be present or absent, and the criteria used to make that determination can strongly affect estimates of the prevalence of dementia [11]. Diagnostic criteria for dementia in ADAMS and CHAP were found to differ. In ADAMS, the diagnosis of dementia was on the basis of clinical judgment anchored by the criteria of the Diagnostic and Statistical Manual of Mental Disorders (DSM) III-R and IV [12,13], whereas the diagnosis in CHAP was on the basis of clinical judgment anchored by the criteria of the National Institutes of Neurological and Communicative Disorders and Stroke and the Alzheimer's Disease and Related Disorders Association (NINCDS-ADRDA [14]). Both criteria require a history of cognitive decline and impairment in multiple cognitive domains. An important difference is that the DSM criteria require the cognitive decline to be of sufficient severity to impair daily function. The latter is typically documented by an interview with an informant. By contrast, in the NINCDS-ADRDA criteria for dementia, evidence of cognitive decline is obtained by medical history from the participant and an informant, if available, with decline documented by cognitive performance testing. The DSM requirement of both functional impairment and evidence of cognitive impairment on neuropsychological tests would most likely identify persons with a greater degree of cognitive impairment as compared with just using performance on neuropsychological tests alone. Therefore, all other factors being equal, more people would be expected to meet NINCDS-ADRDA criteria for dementia than DSM criteria.

\subsection{Separating dementia from normality}

The primary clinical manifestation of dementia is accelerated cognitive decline over the course of many months to years. However, rates of cognitive decline in old age more closely approximate a normal distribution rather than a bimodal one [15]; therefore, it is often not immediately clear where to place the line between normal aging and dementia. One response to this problem has been to create a new syndrome, most commonly referred to as either mild cognitive impairment (MCI) [16] or cognitive impairment not dementia (CIND) [17], for individuals with cognitive impairment not severe enough to warrant a diagnosis of dementia. Although these intermediate syndromes have proven useful, there is no secure agreement on how best to implement diagnostic criteria or distinguish them from dementia. Moreover, to the extent that impairment in daily function is measured with error, that error compounds the measurement error in cognitive assessments.

In ADAMS 241 cases of CIND were identified, which translate to a national estimate of 5.4 million individuals with upweighting. Of this group, the ADAMS assigned a diagnosis of prodromal AD (defined as "a pattern of clinical symptoms or performance on neuropsychological testing suggestive of prodromal AD and no other medical or neuropsychiatric conditions present to preclude an eventual diagnosis of AD" [17]) to 98 individuals (1.9 million individuals with upweighting). Therefore, shifting the threshold for dementia toward that of CIND could account for very large differences in $\mathrm{AD}$ prevalence.

\subsection{Differential diagnosis of dementia}

A diagnosis of $\mathrm{AD}$ requires additional criteria other than the diagnosis of dementia. Both ADAMS and CHAP used NINCDS-ADRDA criteria for AD. These criteria require progressive loss of cognitive abilities, one of which must be memory. The studies differed in how they approached differential diagnosis in the presence of comorbidities and atypical disease presentation.

\subsubsection{Comorbidity}

$\mathrm{AD}$ is widely recognized as the leading cause of dementia, but other pathologic factors contribute to cognitive impairment as well, particularly cerebrovascular disease and Lewy bodies, which can cause vascular dementia and Lewy body disease but, in addition, can also contribute to mixed dementia [18]. However, clinical classification of the cause of dementia is difficult. In fact, communitybased clinical-pathologic studies indicate that most cases of dementia arise from mixed pathologic processes, most commonly $\mathrm{AD}$ pathologic changes (i.e., neuritic plaques, neurofibrillary tangles) in conjunction with cerebrovascular disease and often Lewy bodies; furthermore, most cases of dementia meeting NINCDS/ADRDA criteria for clinical AD arise from mixed pathologic processes [19,20]. Finally, both cortical and subcortical cerebral vascular disease, in addition to $\mathrm{AD}$ pathology, contribute to impaired episodic memory and other domains of cognition suggesting that cognitive profiles have limited utility in separating these common conditions [21,22]. Although cerebrovascular disease is common in the brains of old people with dementia, it is rarely the only pathologic finding. That is, dementia is rarely a result of cerebrovascular disease in isolation. Of 308 individuals with dementia in ADAMS, 48 (16\%) were diagnosed with vascular dementia on the basis of a combination of reported temporally related stroke and cognitive decline, medical record documentation of stroke, and a cognitive profile consistent with cerebrovascular disease. When results were upweighted to the population, these 48 individuals represented approximately 0.59 million affected persons in the United States. By contrast, in CHAP, vascular disease was often recognized as contributing to cognitive impairment in individuals diagnosed with $\mathrm{AD}$. Therefore, persons meeting criteria for both $\mathrm{AD}$ and vascular dementia were included as cases of $\mathrm{AD}$ and pure vascular dementia was rarely diagnosed [23]. 


\subsubsection{Indeterminate dementia}

In some cases, clinical classification of dementia subtype is particularly difficult, usually because of insufficient or conflicting data or atypical presentation. The DSM-IV allows for this possibility with the category of "dementia not otherwise specified" [13]. In ADAMS, 23 of 308 dementia cases $(7 \%)$ received a diagnosis of "dementia, undetermined etiology" when the clinical presentation was too atypical to permit a diagnosis of possible $\mathrm{AD}$, but there was no other apparent cause for dementia. After being upweighted to the population, these 23 individuals represented 0.34 million individuals. In CHAP, the study design required that a minimum of one contributing factor be assigned to all dementia cases. Because AD is by far the most common cause of late life dementia, it is likely that many persons who would have been classified as having dementia of undetermined etiology in ADAMS were classified as AD in CHAP despite meager or conflicting data. This may have slightly inflated the number of AD cases in CHAP.

\section{Characteristics of persons diagnosed differently by different criteria}

We reviewed previously the two major diagnostic differences, the use of the criterion for the presence of functional impairment, identified by an informant, to distinguish dementia from CIND in ADAMS, and the attribution of dementias of primarily vascular or potentially mixed etiology to AD in CHAP, which could account for most of the difference in AD prevalence estimates. In Table 1 we show, using ADAMS data, how these differentially diagnosed groups compare on some key indicators of cognitive function, functional impairment, and genetics.

The bottom row of Table 1 describes persons diagnosed with prevalent AD in ADAMS. Their average scores on the Mini-Mental State Examination and the Dementia Severity Rating Scale indicated moderate dementia; the average Clinical Dementia Rating Scale scores were consistent with mild to moderate dementia. In the two rows above them, are the groups who were identified in ADAMS as demented but with non-AD etiology, and as CIND with prodromal AD. Taken together with the bottom row, these groups add-up to 5.3 million persons, which is slightly higher than the CHAP estimate of AD. It is likely that the CHAP diagnostic criteria would have classified most of the individuals in these three rows as AD. Although the cognitive and functional level of the vascular and other dementia group was quite similar to that of the $\mathrm{AD}$ group, the CIND prodromal AD category had much better global cognitive and functional performance, though it was much worse than the normal group (mean Mini-Mental State Examination score = 24.28, mean Clinical Dementia Rating Scale score = 0.49 ), and delayed recall intermediate as compared with the dementia group and the remaining CIND group (mean $=3.18$ ), which was consistent with the procedures used to classify these persons. We find it interesting that, although the prodromal AD subgroup within the CIND category had cognitive function very similar to CIND cases with other etiologies, their episodic memory was more impaired and the presence of any apolipoprotein E $\varepsilon 4$ allele in this group was nearly three times higher than the other CIND cases and actually higher than the AD dementia group. Moreover, the combined group of vascular and other dementia also had an $\varepsilon 4$ allele frequency comparable with that of the AD dementia group. Although this is a very limited genetic profile, the $\varepsilon 4$ allele is strongly related to AD pathology [24]. Thus, these data suggest that the prodromal AD group may be exhibiting the early signs of cognitive impairment related to the pathology of $\mathrm{AD}$.

\section{Discussion}

Estimating the prevalence of $\mathrm{AD}$ in the United States is a complex process that requires many assumptions and decisions. Therefore, it is not surprising that estimates have previously been noted to be variable [25]. We reviewed several differences between the Hebert et al [3] and Plassman et al [6] studies that could have affected results. In some cases, we were able to quantitatively estimate the effects of different decisions. The results suggest that the difference between the two prevalence estimates could be entirely accounted for by two factors. First, a diagnosis of dementia in ADAMS required functional impairment reported by an informant and as a result was most likely associated with a higher level of cognitive impairment than a dementia diagnosis in CHAP. By lowering the threshold for dementia to

Table 1

Descriptive information from ADAMS weighted to the U.S. population*

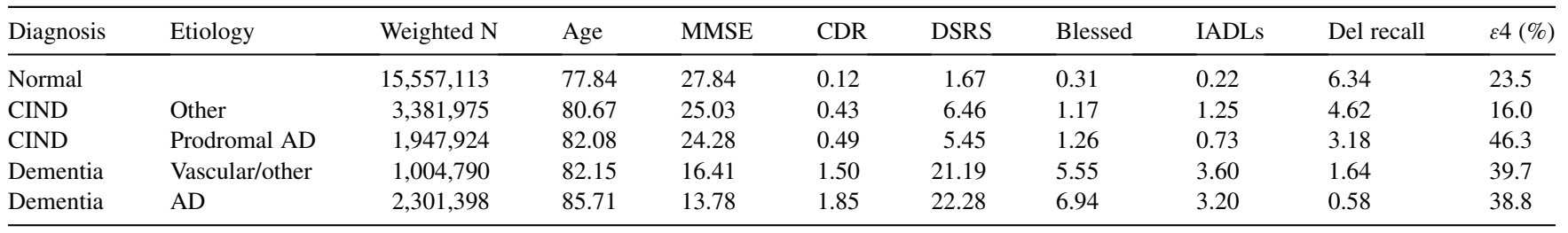

Abbreviations: ADAMS, Aging, Demographics, and Memory study; AD, Alzheimer's disease; CIND, cognitive impairment not dementia; MMSE, MiniMental State Examination; CDR, Clinical Dementia Rating Scale; DSRS, Dementia Severity Rating Scale; Blessed, Blessed Dementia Scale; IADLs, number (of six) of impaired instrumental activities of daily living; $\varepsilon 4$, any apolipoprotein $\mathrm{E} \varepsilon 4$ allele; Del recall, word list recall.

*Weighted using ADAMS sampling weight. 
classify all individuals with prodromal $\mathrm{AD}$ as demented, one could account for a maximum of two million cases and much of the difference between the prevalence estimates. This observation is consistent with previous analyses of the effect of dementia criteria on estimates of its prevalence [11].

A second factor that affected estimates of AD prevalence in the two studies was differential diagnosis. In CHAP, more than $90 \%$ of dementia was classified as AD. This includes those judged to have $\mathrm{AD}$ in addition to one or more other conditions contributing to cognitive impairment such as vascular cognitive impairment. In ADAMS, similar to other epidemiological studies of dementia, a substantial subset of dementia was classified as vascular dementia, which was very rare in CHAP. An additional proportion of dementia cases in ADAMS was classified as etiology unknown, which was not an option in CHAP. Together, these factors could have accounted for a difference of a maximum of 1 million cases in the prevalence estimates.

So which prevalence number is correct-the 2.4 million from ADAMS or the 4.5 million from CHAP? The answer is that the choice of prevalence estimate must be matched to the specific question it is used to answer. For example, if the question is how many people with $\mathrm{AD}$ are dependent in activities of daily living, it seems appropriate to exclude the highly functioning early $\mathrm{AD}$ cases in that count. In fact, such a question was a focus of some early prevalence studies that were restricted to persons with moderate to severe dementia [26,27]. If the question is how many people might benefit from an effective therapy aimed at the underlying pathology of $\mathrm{AD}$, such as an anti-amyloid agent, it seems appropriate to include those persons with mild forms of cognitive dysfunction as a result of the underlying disease pathology. Further, because recent clinical-pathologic studies suggest that $\mathrm{AD}$ pathology and cerebrovascular pathology have additive effects on the odds of dementia and cognitive impairment [19-22], it is possible that persons with mixed pathologies might also benefit from an agent that affects the underlying pathology of $\mathrm{AD}$, thus making it important to identify the contribution of AD pathology to cognition in persons who also meet criteria for vascular dementia.

An important evolving debate is whether the definition of dementia and AD should shift from dependence on a level of cognitive impairment that leads to moderate disability in daily life to much milder levels of cognitive impairment, or even criteria that depend on the presence of biomarkers or imaging findings associated with $\mathrm{AD}$ pathology that may have not yet caused significant cognitive or functional impairment. Many persons had MCI in CHAP [28,29] and CIND in ADAMS [17]. In fact, Plassman et al [17] estimated that 5.4 million people in the United States had CIND in 2002. Recent data suggest that persons with this syndrome have mild limitations in daily living [30] and are at greater risk for cognitive decline [29,31] and death [28,31]. In addition, clinical-pathologic research suggests that many old people without dementia meet pathologic criteria for
AD [20,32-38]. In one study, for example, nearly twothirds of those who died with MCI and one-third of those who died with no apparent cognitive impairment met pathologic criteria for the disease [37]. Further, the correlation of the pathologic findings with cognitive impairment was similar in those with and without dementia. Therefore, it is not surprising that some investigators suggest that many of those with MCI or CIND already have AD [39], or that a recent panel recommended revising $\mathrm{AD}$ criteria to include a subset of MCI with memory impairment supplemented by a biomarker of AD pathology [40]. In fact, the National Institute on Aging and the Alzheimer's Association recently established three working groups to (1) revise the NINCDSADRDA criteria for AD, (2) better define MCI, and (3) define persons with preclinical AD by referring to persons with AD pathology who do not meet clinical criteria for dementia or MCI. Draft reports were presented at the $2010 \mathrm{In}$ ternational Conference on AD, in Honolulu, Hawaii. Thus, persons with dementia may only represent a fraction of those who have the underlying disease pathology and whose cognition and behavior may already show some subtle changes because of this pathology. Although there are ongoing efforts to make the diagnosis of $\mathrm{AD}$ even earlier in the disease process, in the absence of clearer evidence of which cognitively impaired individuals will progress to dementia and of robust therapy for the treatment or prevention of cognitive decline, one should also be cautious about the potential risks and "clinical cascade" that might result from labeling as "disease" pathologic changes in the brain or subtle cognitive changes that might not affect the daily lives, or life expectancy, of older adults [41].

\section{Acknowledgments}

This research was supported by National Institute on $\mathrm{Ag}$ ing grants R01AG17917, R01AG11101, U01AG09740, and R01AG027010.

\section{References}

[1] Brookmeyer R, Gray S, Kawas C. Projections of Alzheimer's disease in the United States and the public health impact of delaying disease onset. Am J Public Health 1998;88:1337-42.

[2] Sloane PD, Zimmerman S, Suchindran C, Reed P, Wang L, Boustani M, et al. The public health impact of Alzheimer's disease, 2000-2050: potential implication of treatment advances. Ann Rev Public Health 2002;23:213-31.

[3] Hebert LE, Scherr PA, Bienias JL, Bennett DA, Evans DA. Alzheimer disease in the US population: prevalence estimates using the 2000 census. Arch Neurol 2003;60:1119-22.

[4] Evans DA. Estimated prevalence of Alzheimer's disease in the United States. Milbank Q 1990;68:267-89.

[5] United States General Accounting Office. Alzheimer's disease: estimates of prevalence in the United States. Washington, DC: United States General Accounting Office; 1998.

[6] Plassman BL, Langa KM, Fisher GG, Heeringa SG, Weir DR, Ofstedal MB, et al. Prevalence of dementia in the United States: the aging, demographics, and memory study. Neuroepidemiology 2007; 29:125-32. 
[7] Juster FT, Suzman R. An overview of the health and retirement study. J Hum Resour 1995;30(Suppl):S7-S56.

[8] Ferri CP, Prince M, Brayne C, Brodaty H, Fratiglioni L, Ganguli M, et al. Global prevalence of dementia: a Delphi consensus study. Lancet 2005;366:2112-7.

[9] Hirtz D, Thurman DJ, Gwinn-Hardy K, Mohamed M, Chaudhuri AR, Zalutsky R. How common are the "common" neurologic disorders? Neurology 2007;68:326-37.

[10] Wolfson C, Wolfson DB, Asgharian M, M'Lan CE, Østbye T, Rockwood K, et al. A reevaluation of the duration of survival after the onset of dementia. N Eng J Med 2001;344:1111-6.

[11] Erkinjuntti T, Østbye T, Steenhuis R, Hachinski V. The effect of different diagnostic criteria on the prevalence of dementia. N Engl J Med 1997;337:1667-74.

[12] American Psychiatric Association. Diagnostic and Statistical Manual of Mental Disorders. 3rd ed. Washington, DC: American Psychiatric Association; 1987.

[13] American Psychiatric Association. Diagnostic and Statistical Manual of Mental Disorders. 4th ed. Washington, DC: American Psychiatric Association; 1994.

[14] McKhann G, Drachman D, Folstein M, Katzman R, Price D, Stadlan E. Clinical diagnosis of Alzheimer's disease: report of the NINCDS/ ADRDA work group under the auspices of Department of Health and Human Services Task Force on Alzheimer's disease. Neurology 1984;34:939-44.

[15] Wilson RS, Beckett LA, Barnes LL, Schneider JA, Bach J, Evans DA, et al. Individual differences in rates of change in cognitive abilities of older persons. Psychol Aging 2002;17:179-93.

[16] Peterson RC, Smith GE, Waring SC, Ivnik RJ, Tangalos EG, Kokmen E. Mild cognitive impairment. Arch Neurol 1999; $56: 303-8$

[17] Plassman BL, Langa KM, Fisher GG, Heeringa SG, Weir DR, Ofstedal MB, et al. Prevalence of cognitive impairment without dementia in the United States. Ann Int Med 2008;148:427-34.

[18] Langa KM, Foster NL, Larson EB. Mixed dementia: emerging concepts and therapeutic implications. JAMA 2004;292:2901-8.

[19] Schneider JA, Arvanitakis Z, Leurgans SE, Bennett DA. The neuropathology of probable Alzheimer disease and mild cognitive impairment. Ann Neurol 2009;66:200-8.

[20] Sonnen JA, Larson EB, Crane PK, Haneuse S, Li G, Schellenberg GD, et al. Pathological correlates of dementia in a longitudinal, populationbased sample of aging. Ann Neurol 2007;62:406-13.

[21] Schneider JA, Wilson RS, Bienias JL, Evans DA, Bennett DA. Cerebral infarctions and the likelihood of dementia from Alzheimer's disease pathology. Neurology 2004;62:1148-52.

[22] Schneider JA, Boyle PA, Arvanitakis Z, Bienias JL, Bennett DA. Subcortical cerebral infarcts, episodic memory, and AD pathology in older persons. Ann Neurol 2007;62:59-66.

[23] Evans DA, Bennett DA, Wilson RS, Bienias JL, Morris MC, Scherr PA, et al. Incidence of Alzheimer disease in a biracial urban community: relation to apolipoprotein E allele status. Arch Neurol 2003;60:185-9.
[24] Bennett DA, De Jager PL, Leurgans SE, Schneider JA. Neuropathologic intermediate phenotypes enhance association to Alzheimer susceptibility alleles. Neurology 2009;72:1495-503.

[25] Corrada M, Brookmeyer R, Kawas C. Sources of variability in prevalence rates of Alzheimer's disease. Int J Epidemiol 1995;24:1000-5.

[26] Schoenberg BS, Anderson DW, Haerer AF. Severe dementia: prevalence and clinical features in a biracial US population. Arch Neurol 1985;42:740-3.

[27] Bachman DL, Wolf PA, Linn R, Knoefel JE, Cobb J, Belanger A, et al. Prevalence of dementia and probable senile dementia of the Alzheimer type in the Framingham study. Neurology 1992;42:115-9.

[28] Wilson RS, Aggarwal NT, Barnes LL, Bienias JL, Mendes de Leon CF, Evans DA. Biracial population study of mortality in mild cognitive impairment and Alzheimer disease. Arch Neurol 2009;66:767-72.

[29] Wilson RS, Aggarwal NT, Barnes LL, Mendes de Leon CF, Hebert LE, Evans DA. Cognitive decline in incident Alzheimer disease in a community population. Neurology 2010;74:951-5.

[30] Perneczky R, Pohl C, Sorg C, Hartmann J, Komossa K, Alexopoulos P, et al. Complex activities of daily living in mild cognitive impairment: conceptual and diagnostic issues. Age Ageing 2006;35:240-5.

[31] Bennett DA, Wilson RS, Schneider JA, Evans DA, Beckett LA, Aggarwal NT, et al. Natural history of mild cognitive impairment in older persons. Neurology 2002;59:198-205.

[32] Riley KP, Snowdon DA, Markesbery WR. Alzheimer's neurofibrillary pathology and the spectrum of cognitive function: findings from the Nun Study. Ann Neurol 2002;51:567-77.

[33] Galvin JE, Powlishta KK, Wilkins K, McKeel DW, Xiong C, Grant E, et al. Predictors of preclinical Alzheimer disease and dementia: a clinicopathologic study. Arch Neurol 2005;62:758-65.

[34] Petersen RC, Parisi JE, Dickson DW, Johnson KA, Knopman DS, Boeve BF, et al. Neuropathologic features of amnestic mild cognitive impairment. Arch Neurol 2006;63:665-72.

[35] Markesbery WR, Schmitt FA, Kryscio RJ, Davis DG, Smith CD, Wekstein DR. Neuropathologic substrate of mild cognitive impairment. Arch Neurol 2006;62:38-46.

[36] Driscoll I, Resnick SM, Troncoso JC, An Y, O’Brien R, Zonderman AB. Impact of Alzheimer's pathology on cognitive trajectories in nondemented elderly. Ann Neurol 2006;60:688-95.

[37] Bennett DA, Schneider JA, Arvanitakis Z, Kelly JF, Aggarwal NT, Shah RC, et al. Neuropathology of older persons without cognitive impairment from two community-based studies. Neurology 2006;66:1837-44.

[38] Bennett DA, Schneider JA, Bienias JL, Evans DA, Wilson RS. Mild cognitive impairment is related to Alzheimer's disease pathology and cerebral infarctions. Neurology 2005;64:834-41.

[39] Morris JC. Mild cognitive impairment is early-stage Alzheimer disease: time to revise diagnostic criteria. Arch Neurol 2006;63:15-6.

[40] Dubois B, Feldman HH, Jacova C, DeKosky ST, Barberger-Gateau P, Cummings J, et al. Research criteria for the diagnosis of Alzheimer's disease: revising the NINCDS-ADRDA criteria. Lancet Neurol 2007; 6:734-46.

[41] Larson EB, Langa KM. The rising tide of dementia worldwide. Lancet 2008;372:430-2. 